\title{
MEMS-based phased arrays for Army applications
}

Paul Ruffin, James Holt, James Mullins, Tracy Hudson, Janice Rock

Paul B. Ruffin, James C. Holt, James H. Mullins, Tracy Hudson, Janice Rock, "MEMS-based phased arrays for Army applications," Proc. SPIE 6528, Nanosensors, Microsensors, and Biosensors and Systems 2007, 652802 (11 April 2007); doi: 10.1117/12.721760 Health Monitoring, 2007, San Diego, California, United States 


\title{
MEMS-based Phased Arrays for Army Applications
}

\author{
Paul B. Ruffin, James C. Holt, James H. Mullins, Tracy Hudson, Janice Rock \\ U. S. Army Research, Development, and Engineering Command \\ ATTN: AMSRD-AMR \\ Redstone Arsenal, Alabama 35898 \\ E-mail: paul.ruffin@us.army.mil \\ Phone: 256-876-8333
}

\begin{abstract}
The Army Aviation and Missile Research, Development, and Engineering Center (AMRDEC) initiated a research and development project several years ago to develop Micro Electro-Mechanical Systems (MEMS)-based phased arrays to provide rapid beam steering for sensors, optical and Radio Frequency (RF) missile seekers, and RF communication links. In particular, the joint AMRDEC/Army Research Laboratory (ARL) project, which leverages low-cost phased array components developed under the Defense Advanced Research Projects Agency (DARPA) Low Cost Cruise Missile Defense (LCCMD) project, is developing RF switches, phase shifters, and passive phased sub-arrays to provide a fast scanning capability for pointing, acquisition, tracking, and data communication; and rugged, optical MEMS-based phased arrays to be employed in small volume, low-cost Laser Detection and Ranging (LADAR) seekers. The current status of the project is disclosed in this paper. Critical technical challenges, which include design and fabrication of the RF switches and phase shifters, design and fabrication of micro lens arrays, control of beam steering, scanning angular resolution and array losses, are discussed. Our approach to overcoming the technical barriers and achieving required performance is also discussed. Finally, the validity of a MEMS technology approach against competing low cost technologies is presented.
\end{abstract}

KEYWORDS: RF and Optical Beam Steering, MEMS-Based Phased Arrays, RF MEMS Phase Shifters, Micro Lens Arrays

\section{INTRODUCTION}

It is well known that phased array antennas, which consist of an array of antenna or phase elements without particular directivity, are used in land vehicle satellite communications. ${ }^{1}$ This subject is well documented in Ref. [2] for microwave and radio frequencies. Focusing and shifting of the radiated energy can be achieved within microseconds using miniature phase shifters or time delay devices. The advantages of phased array radars include power management and efficiency, reliability, rapid beam steering, and good signal reception. However, phased array radar designs, which require several thousands of phase shifters and drivers, are very large, complex, and expensive. This is largely due to the requirement for multiple antennas and elaborate power switching networks to achieve communications over 360 degrees. A large phased array radar sited in Alaska, which is used as part of a Ballistic Missile Early Warning System to monitor the northern sky, is shown in Fig. 1.

Airborne weapon systems currently use large, heavy, and expensive gimbaled telescopes to scan missiles target-acquisition footprints as they fly down range. Smaller and more agile missiles operating in nonconventional warfare will require increased scanning speed and reduced scanning footprints and power consumption. The gimbaled horn antennas make fast scanning and rapid scene correlation for target acquisition and identification, required for seekers aboard fast moving tactical missiles or unmanned aerial vehicles (UAVs), difficult to be accomplished. Phased array antennas are ideal for use in sensors and communication systems used in these airborne platforms to provide early warning of attack, target

Nanosensors, Microsensors, and Biosensors and Systems 2007, edited by Vijay K. Varadan, Proc. of SPIE Vol. 6528, 652802, (2007) · 0277-786X/07/\$18 - doi: 10.1117/12.721760 


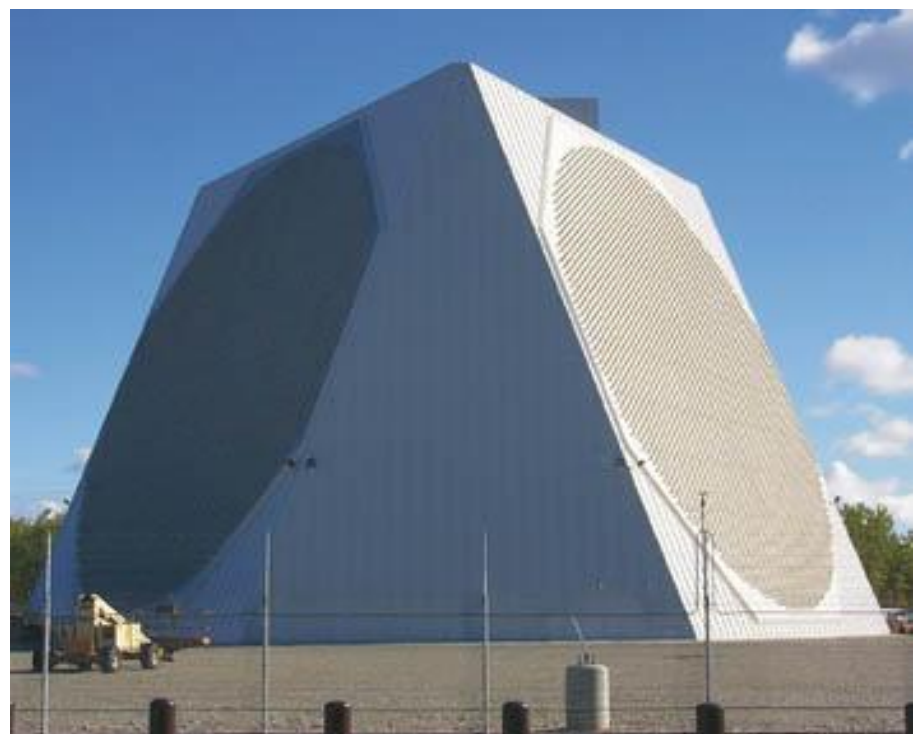

Fig. 1. A Giant Phased Array Radar in Alaska (Courtesy of Alaska District, this 90-foot (27m) diameter radar installation monitors the northern sky. Its construction was part of the Clear Radar Upgrade to make all Ballistic Missile Early Warning System radars phased-array rather than mechanical.) detection and identification, target tracking, and countermeasure decision. ${ }^{3}$ A typical phased array antenna with pod for mounting underneath aircraft wing is shown in Fig. 2.

Electronic Scanning Antennas (ESA) have been considered for airborne weapon systems. The conventional ESA technologies work well for lowfrequency RF (less than K-band) applications. However, when these phased array technologies are considered for tactical missile seekers and disposable airborne communication systems, they become cost prohibitive. Missile seekers tend to use the higher frequencies due to the need for small beam-widths and adequate angular resolution in limited space. Cost increases for these higher frequencies ( $\mathrm{Ku}$ and $\mathrm{Ka}$-band) because of the increased number of antenna elements required to maintain the optimal spacing to eliminate grating

lobes for +/- 90 degrees of RF beam scan.

The Army Aviation and Missile Research, Development, and Engineering Center (AMRDEC) initiated a research and development project several years ago to address the issues associated with rapid beam steering at high frequencies. The joint AMRDEC/Army Research Laboratory (ARL) project, which leverages low-cost phased array components developed under the Defense Advanced Research Projects Agency (DARPA) Low Cost Cruise Missile Defense (LCCMD) project, is developing RF switches, phase shifters, and passive phased sub-arrays to provide a fast scanning capability for pointing, acquisition, tracking, and data communication; and rugged, optical MEMS-based phased arrays to be employed in small volume, low-cost LAser Detection and Ranging (LADAR) seekers. The primary objective of the project is to replace horn antennas with low cost MEMS-based or alternative low cost phased arrays to take advantage of rapid beam pointing without mechanical manipulation. The RF portion of the research is investigating both electrostatic and piezoelectric actuated RF MEMS switches to be used in phased arrays to provide missile seeker capability with near

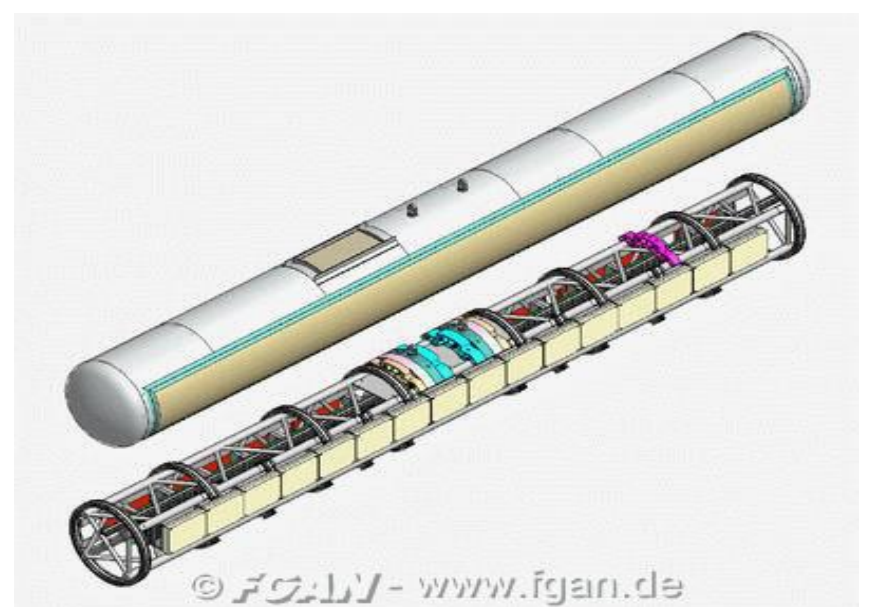

Fig. 2. Pod with phased array antenna of the experimental radar system PAMIR 256, to be mounted underneath aircraft wing (Ref. 4) instant beam steering, selective beam pointing, and multiple target track allowing rapid scene correlation and to provide $360^{\circ}$ coverage with few antennas and directed data transfer with sharpened beams for longer communication ranges with immunity 
to interferences. The optical portion of the research is made possible by the progress in optical MEMS, as discussed in details elsewhere Ref. [5]. A LADAR seeker based on optical MEMS would be extremely attractive due to its wide bandwidth to provide large field of regard and autonomous target acquisition and recognition. Although the propagation of visible light, in general, and the infrared, in particular, can be impeded by weather conditions, the shorter ranges in tactical missile systems alleviate the severity of the problem. In general, the major contribution of optical MEMS to LADAR is to replace the mechanical steering with phased array technology. This paper is intended to present the status of the AMRDEC research and development project, which is developing both RF and optical phased arrays to replace mechanical beam steering and stability mechanisms for more precise and rapid scanning capability.

The paper is arranged as follows. A brief discussion of rapid beam scanning by a missile seeker is provided in Section 2. The status of research efforts to develop RF switches, phase shifters, and MEMSbased phased arrays for missile communication links and seekers are presented in Section 3. Recent work and some technical challenges associated with micro lens arrays (MLA) designed for LADAR seekers are discussed in Section 4. A laser pulse repetition frequency enhancer, which permits ultra-high speed beam scanning, is discussed in Section 5. A summary is provided in Section 6.

\section{RAPID BEAM SCANNING}

Air and space-borne radar systems are well suited for wide area surveillance for moving vehicles. An illustration of a missile seeker acquisition scan footprint is shown in Fig. 3. The seeker scans a footprint of $80 \mathrm{~m} \times 108 \mathrm{~m}$ in 0.75 seconds at a slant range of $648 \mathrm{~m}$. The airframe searches targets at a depression angle of 18 degrees. The air velocity of the system is approximately $80 \mathrm{~m} / \mathrm{s}$ while traveling at an altitude greater than or equal to 200 meters. A scanner response time on the order of $100 \mu \mathrm{sec}$ is required for such a scenario.

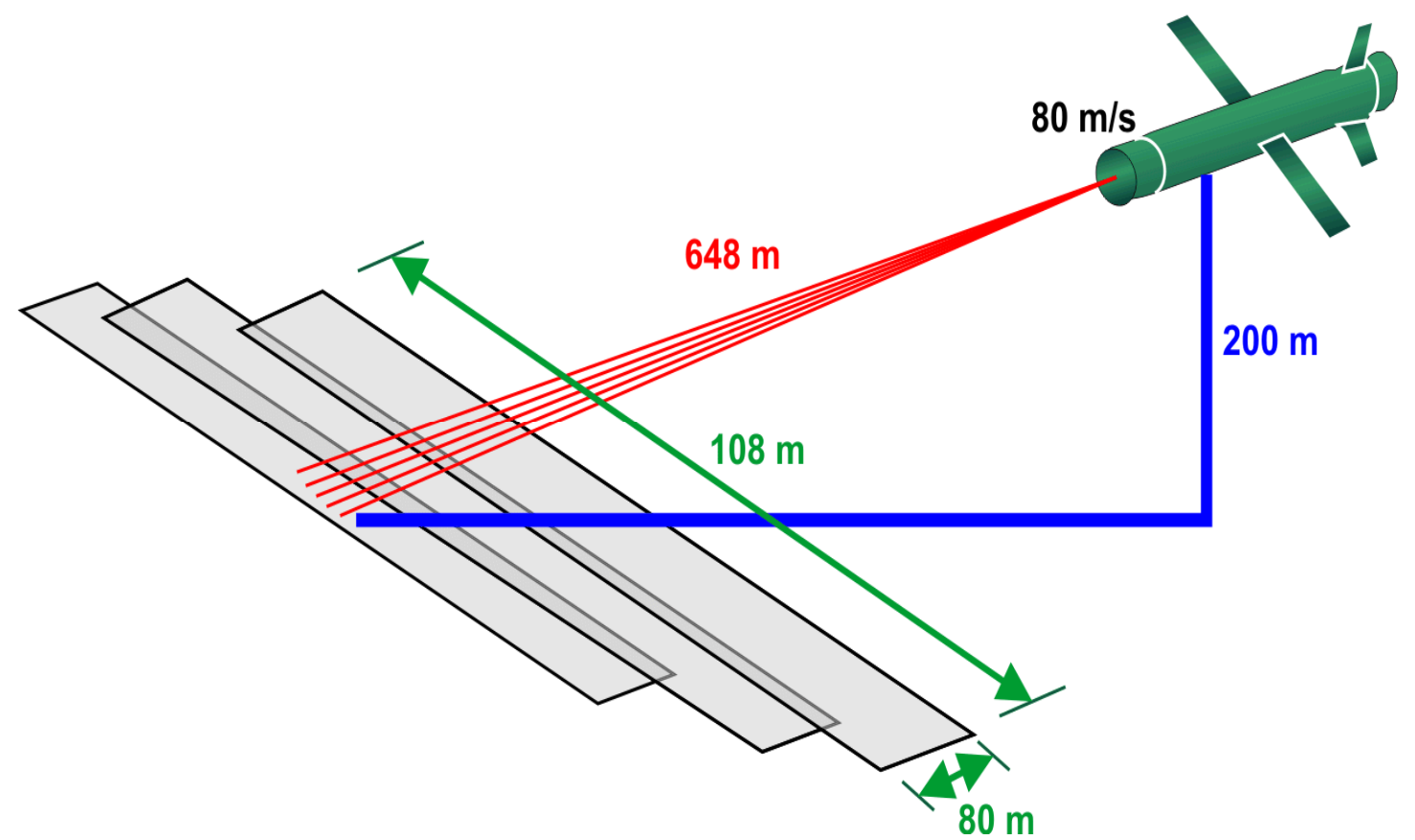

Fig. 3. An illustration of a missile seeker acquisition scan footprint

The tracking accuracy of the missile RF seeker depends on the magnitude of the signal-to-noise ratio $(\mathrm{S} / \mathrm{N})$. The conventional form for $\mathrm{S} / \mathrm{N}$, from the radar equation, is ${ }^{6}$ 


$$
S / N=\left[\frac{P}{(4 \pi)^{3}} /(k T B L)\right] *\left\{G^{2} \lambda^{2} * \frac{(\text { Radar Cross Section })}{R^{4}}\right\}
$$

where $\mathrm{kT} * \mathrm{~B}$ is the product of noise (k-Boltzman's constant and T-temperature) and noise bandwidth, $\mathrm{B}$, in units of energy/sec. $L$ is loss due to propagation through the atmosphere. $G=4 \pi \mathrm{A}_{\text {ant }} / \lambda^{2}$ is the gain factor and $\mathrm{R}$ is the range of the radar. $\mathrm{A}_{\text {ant }}$ is the antenna area.

The conventional expression is modified for a LADAR seeker using direct measurement for which the band noise $\mathrm{kT}^{*} \mathrm{~B}$ is replaced by $(\mathrm{A} * \mathrm{~B})^{1 / 2} / \mathrm{D}_{\mathrm{d}}$, where $\mathrm{D}_{\mathrm{d}}$ is the detectivity in the units of $\mathrm{cm}-\mathrm{Hz} \mathrm{z}^{1 / 2} / \mathrm{Watt}$ and $\mathrm{A}$ is the detector area. The radar cross section becomes the collection aperture of the LADAR.

Eq. 1 shows that $\mathrm{S} / \mathrm{N}$ decreases drastically $\left(1 / \mathrm{R}^{4}\right)$ as range increases. Doubling the slant range of the missile reduces $\mathrm{S} / \mathrm{N}$ by a factor of 16 or by $94 \%$. Rapid beam scanning is required in order for missile communication links and seekers to provide an increased ability to track and engage multiple fleeting targets at increased range. Efforts are underway to develop low-cost phased array components and designs, sub-arrays demonstrating RF and optical capabilities, and integrated sub-arrays/phased arrays, which will enable seekers and communications links to operate more quickly while tracking an increased number of targets at increased range with no cost increase.

RF and optical MEMS, two unrelated areas, both potentially can have a positive impact on future designs of seekers and communication devices for missiles and unmanned aerial vehicles. Fig. 4 illustrates how a conventional gimbaled horn antenna can be replaced with a 2-dimensional optical phased array scanner in the case of a LADAR Seeker and a passive phased array in the case of a RF Seeker.

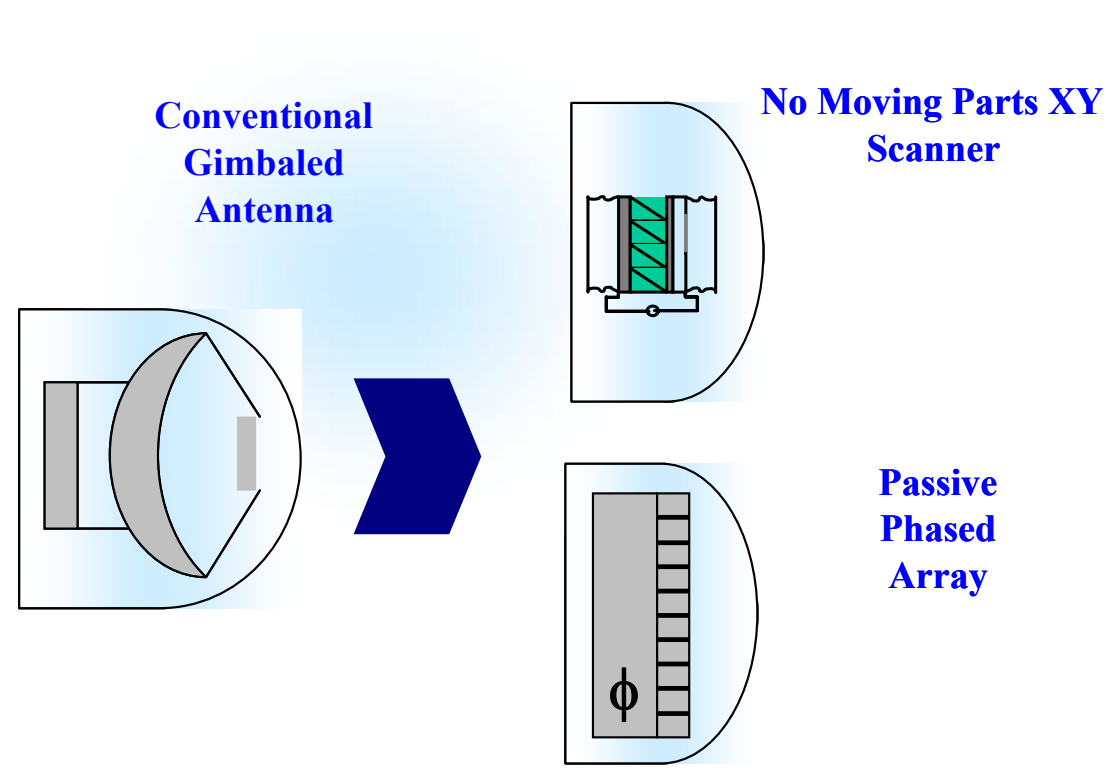

Fig. 4. An illustration of a conventional gimbaled seeker antenna being replaced by optical and RF phased arrays

Micro Electro-Mechanical Systems (MEMS) switches are ideal for use in applications such as radar systems for defense applications, automation radars, satellite communication systems, wireless communication systems, instrumentation systems, etc. MEMS-based switches, which have excellent 
performance at microwave to millimeter wave frequencies, offer a number of advantages over traditional semiconductor switches such as GaAs-based FETs, PHEMT or PIN-diode switches, etc. ${ }^{78,9,10}$ Advantages of MEMS-based RF switches include: low insertion loss $(0.1 \mathrm{~dB})$ and high isolation $(<-30 \mathrm{~dB})$, small size and weight, low power consumption (10-100 $\mathrm{nJ} /$ cycle), high bandwidth, very high signal linearity (IP3 $>80$ $\mathrm{dBm}$ ), very low inter-modulation distortion, ease of integration, etc. Disadvantages of RF MEMS-based switches include: high actuation voltage, switching time (10's $\mu \mathrm{sec})$, low power handling $(50-500 \mathrm{~mW})$, cycle reliability, packaging difficult and costly, overall cost, etc. In addition, optical MEMS phased arrays offer advantages over liquid crystals and other micro optical circuits when the required response time is pushed to less than $0.1 \mathrm{msec}$, which approaches the ultimate limit of liquid crystals. Micro lens arrays are considered for the 2-D optical scanner used in a LADAR seeker. The status of the research efforts at AMRDEC to develop MEMS-based RF switches, phased shifters, micro lens arrays, and sub-arrays to replace gimbals in RF seekers and LADAR seekers is presented in the remaining sections of this paper.

\section{RF MEMS-BASED PHASED ARRAYS}

Mullins provided details of the advantages of RF phased array antennas over gimbaled systems in Ref. [11]. The phased arrays can reposition a beam in an insignificant amount of time. The rapid repositioning of the beam can allow for the tracking of multiple targets while seeking new targets. A conceptual representation of

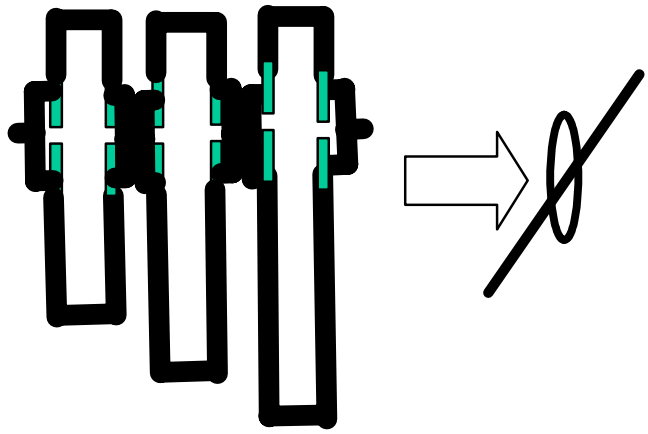

Fig. 5. Conceptual 3 bit phase shifter based on MEMS switches a 3 bit phase shifter, based on MEMS switches is shown in Fig. 5. The phase shifter is enabled by the MEMS-based switches opening and closing the paths for electromagnetic energy from the input to the output. The top path of the phase shifter, illustrated in Fig. 5, represents the reference ( 0 or 360 degree) phase shift. The lower path provides for $45,90,180$, and a combination of these. This 3 bit phase shifter design requires $12 \mathrm{MEMS}$ switches to provide phase shifts of 0 (360), 45, 90, 135, $180,225,270$, and 315 degrees. ${ }^{11}$

The contact type of RF MEMS switches, which produces a closed or open circuit in the RF transmission line via mechanical movement, can vary from capacitive (membrane) to ohmic (metal-metal). The required forces for the mechanical movement are enabled via electrostatic, piezoelectric, thermal, or magneto-static actuation. Electrostatic type switches are used in a variety of applications, such as mobile phones. One of the drawbacks of the electrostatic switch is high actuation voltage. One of the drawbacks of the thermal switch is high power consumption.

Low cost piezoelectric and electrostatic actuated MEMS switches are investigated under the AMRDEC research project ${ }^{11,12}$ Fig. 6 shows a typical cantilever type MEMS switch. The cantilever beam consists of a metal beam suspended above a control electrode (gate). The base of the beam is anchored to a signal electrode (source) and the free standing tip of the beam is suspended above a third electrode (drain). The actuation voltage required to open and close the switch can be applied by electrostatic or piezoelectric actuation. When a sufficient enough voltage is applied to the gate

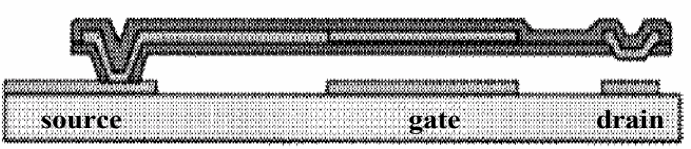

(a)

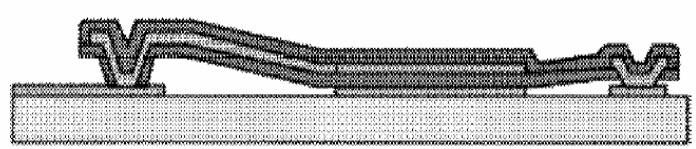

(b)

Fig 6. Cantilever-Type MEMS Switch in Open and Closed Positions relative to the source the beam is pulled down toward the drain until the signal tip and the drain contacts come together as shown in Fig. 6b. At that point 


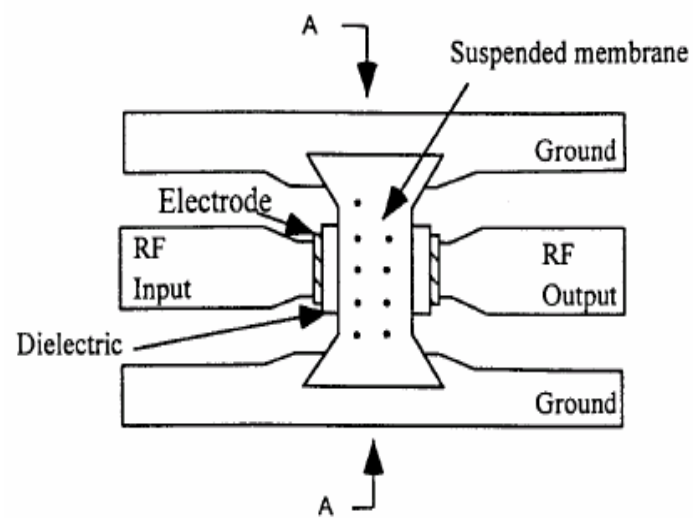

Fig 7. Top View of Capacative Type MEMS Switch

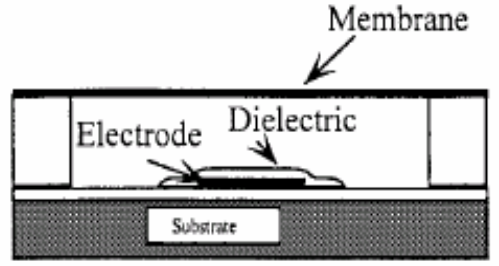

(a)

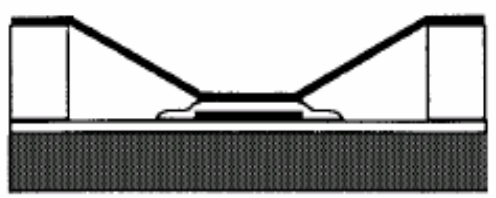

(b)

Fig 8. Side View of Capacative-Type MEMS Switch in Open (a) and Closed (b) Positions

the switch is closed forming a signal path through the beam. When the switch is closed (on state), there is metal-metal contact as shown in Fig. 6.

Fig. 7 shows a top view of a capacitive (Shunt) type MEMS switch. An end view is shown in Fig. 8. The switch is opened and closed via electrostatic actuation. When the switch is closed (on state) as shown in Fig. 8b, there is metal-insulator-metal contact.

The electrostatic force between the top and bottom electrodes in Fig. 8 can be expressed as ${ }^{7,8}$

$$
\mathrm{F}=\varepsilon_{\mathrm{a}} \mathrm{AV}^{2} /\left[2\left(\mathrm{~h}_{\mathrm{a}}+\mathrm{t}_{\mathrm{d}} / \varepsilon_{\mathrm{r}}\right)^{2}\right]
$$

where $\varepsilon_{a}$ is the dielectric constant of air, $A$ is the area of the electrode, $V$ is the voltage, $h_{a}$ is the air gap between the dielectric and the membrane, $t_{d}$ is the thickness of the dielectric layer, and $\varepsilon_{r}$ is a relative dielectric constant. Typical values for $\mathrm{F}$ range from 100 to $200 \mu \mathrm{N}$.

Passive phased sub-arrays are being developed in the laboratory using both piezoelectric and electrostatic actuated switches in phase shifters. More details for the MEMS-based phased arrays used in the Ku-band communication link and the Ka-band missile seeker are provided in the next two subsections.

\subsection{RF Communication Link}

RF MEMS communications links are used in missile systems to provide rapid transmit and receive telemetry data, as well as imagery of diverse objects on the battlefield. Fig. 9 shows a conceptual implementation of phased array antennas used in a communications link for a non-recoverable surveillance system.

In the AMRDEC project, a 2-inch aperture, which encompasses 28 antenna or phase elements, is selected for the Ku-band communication link. A 2-bit phase shifter can be used in a phased array for a Ku-band communication link. A MEMS-based phase array for a $\mathrm{Ku}$ band communications link can provide directional beam steering to reduce the

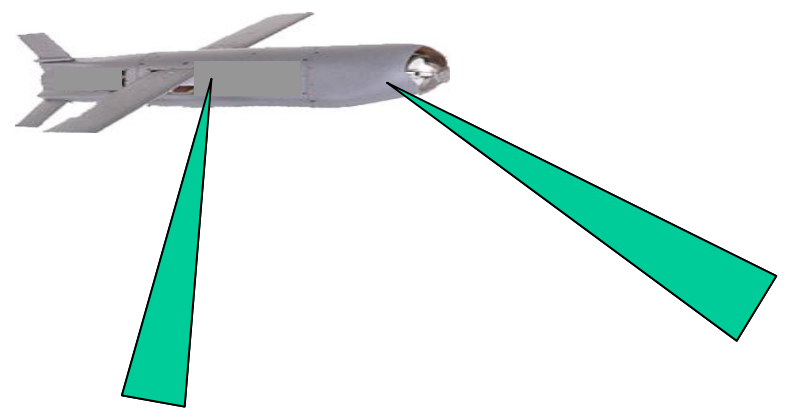

Fig 9. Phased Array Antennas Used in Communication Links. 
susceptibility to jamming, as well as provide for a longer communications range with lower transmitted power. ${ }^{11,12}$

The technical challenges for the design of a Ku-band MEMS-based phase array for a communications link fall in the areas of reliability, packaging, and low-actuating voltages. In the reliability area, stiction due to dielectric charging is overcome with improved design of dielectric materials, metal contact force is increased with piezoelectrics, metal contact degradation is overcome using various alloys, and moisture failure mechanism is overcome with hermetic packaging. In the packaging area, losses experienced with leaded package carriers are overcome with on-wafer packaging techniques. In the area of low-actuating voltages, the actuating voltage issues are improved via switch geometry design improvements. Piezoelectric structures offer a low-actuating alternative. Engineering trades between actuating voltages, reliability and performance (speed and high frequency RF operations), good surface contacts to reduce insertion loss, increased isolation, etc. are being conducted.

Active Electronically Steered Array (AESA) development using commercial fabrication processes has greatly matured over the past several years, reducing size, weight, losses, and individual component losses through higher levels of component integration with fewer packages. Design efforts at AMRDEC for MEMS switches, as well as MEMS-based and Monolithic Microwave Integrated Circuit (MMIC)-based phase shifters, include design of experiment fabrication for Ku-band RF MEMS switches and phase shifters and the fabrication, assembly, and demonstration of two 8-element linear sub-arrays of alternate designs. Significant milestones were achieved via demonstrating RF beam steering achieving $+/-30^{\circ}$ in one design and $+55^{\circ}$ in the second design. Future efforts include: wafer level and far-field/compact range evaluations of MEMS based components leading to the development of a low-loss MEMS-based sub-array and testing of the sub-arrays in the laboratory.

\subsection{RF Seeker}

An airborne or missile seeker radar is used to survey or image a background scene which contains multiple features and objects. MEMS-based phased arrays provide the capability for a missile seeker to acquire and track multiple targets. The missile seeker is a relatively simple system and replacement of the bulky gimbals with RF MEMS-based phased arrays is achievable. Scene matching techniques can be employed in which a surveillance or fire control sensor can provide the missile seeker a scene that contains the most desired target. This multiple target track and scene match capability allows the missile seeker to correlate the input data with the measured

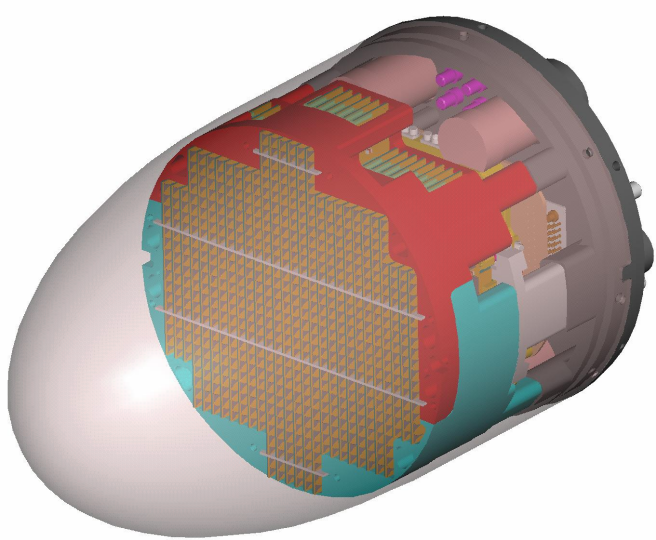

Fig 10. Cutaway View of a Missile Nose Cone Showing an ESA Antenna. scene to capture the desired target.

Fig. 10 shows a cutaway view of a missile nose cone containing an ESA antenna. A 6-inch aperture, which encompasses 1000 antenna or phase elements, for the Ka-band seeker. The 3 bit phase shifter, discussed earlier, can be used in a phased array for a Ka-band missile seeker.

The design challenges discussed in the previous section apply for phased arrays for the RF Seeker. RF MEMS devices including switches, tunable capacitors, high Q inductors, filters and high-Q mechanical resonators are currently available as building blocks for a radar system. The technical challenge is to advance these devices to the operational components level for a viable system. 
Both electrostatic- and piezoelectric-actuated RF MEMS switches for the Ka-band seeker implementation have been designed, fabricated, and tested. In addition, Ka-band MEMS phase shifter architectures have been designed. Ka-band non-steered 8-element sub-array has been designed, fabricated, and tested. Improved design iterations are on-going. Future efforts include: high levels of component integration, heat diffusion and system interconnects investigations, and integration of low-loss, low-cost MEMS-based phase shifters to achieve multiple-elements/single Transmitter/Receiver module design.

\section{OPTICAL MEMS-BASED PHASED ARRAYS}

A LADAR Seeker is particularly attractive because of its ability to provide large field-of-regard and autonomous target acquisition for reconnaissance mission applications. A conventional LADAR seeker is composed of a telescope, which is controlled in azimuth by gimbals. A 2-D scanner, such as the cascaded micro lens array (MLA) illustrated in Fig. 11, is considered to replace the telescope. The MLA implementation allows large angle steering with small motion. The 2-D scanner ensures proper transmission of the laser beam to the target. The cascaded micro-lenses permit laser beam steering in both azimuth and elevation.

Agile beam steering can be accomplished via the cascade of MLAs, which are decentered with respect to each other. Watson presented a theoretical analysis of beam steering with decentered MLAs in Ref. [13]. The results of the analysis suggest that the output of the arrays is analogous to a blazed grating. The angle of the blaze depends on the

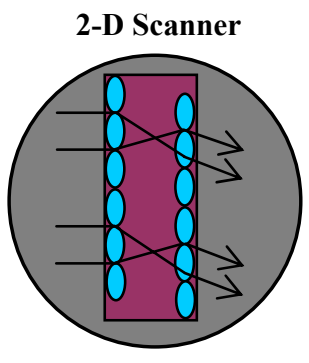

Microlens Array

Fig. 11. Cascaded microlens array amount of the decenter. The allowed steering directions are limited to discrete angles. The amount of energy in the desired steering direction is limited due to the periodic nature of the wavefronts that depend on the fill factor at the output for all steering angles. A lens triplet is needed to obtain the required field of regard for our application. A field lens is incorporated between the front and back lens to reduce light scattering and permit large steering angles.

Beam steering is a function of $\mathrm{f} / \#$, which depends on lens diameter and focal length. Low $\mathrm{f} / \#$ 's (fast lenses) are needed for the MLA configuration selected in our research project, which requires the focal lengths to be external to the MLA substrate while permitting real wafer thickness. The field lens, which has to be $1 / 2$ the $\mathrm{f} / \#$ of the front and back lenses, is limited to $f / 2$. Grayscale microlithography provides low $f / \#$, deep sags, and specific conics. In addition, higher index materials allow for faster lenses with small etch depth.

The challenges for the implementation of MLAs include the optimal method to decenter MLAs with respect to each other in an agile manner, tolerance analysis to determine the specifications (repeatability of focal length, sag depth, conics, etc.), diffraction effect on the output wavefront (aberrations, etc.), optimal manufacture method for MLAs, mechanical fixture for a triplet MLA design, alignment, material index for optimal f/\# effect, limitation of scanning angle, etc. High tolerance MLAs are required for our application.

Current research efforts at AMRDEC consist of the investigation of issues associated with uniformity and fill factor of the micro lenses across the array. Potential error sources can be introduced when constructing low f/\#, tightly packed, small-pitch micro lenses. An analytical study was performed by scientists and engineers at AMRDEC, Stanley Associates, and Wright Patterson Air Force Base to evaluate the magnitude of possible error sources. The initial study addressed the issues of manufacturing processes and production equipment tolerances affect on the performance of micro lens arrays. ${ }^{14}$ It was found that higher aberration and lower coherence occur at larger beam angles. Also, larger diameter micro-lenses, which are more difficult to implement, improve diffraction efficiency and coherence of the system. 
Custom MLAs have been designed and fabricated for use in a non-mechanical beam steering demonstrator. In addition, a non-mechanical prototype beam steering system with a matched set of optics that implements computer controlled piezo-actuators has been assembled. A significant milestone for the optics portion of the AMRDEC research project has been achieved via the demonstration of decentered lens, laser beam steering, achieving $+/-25^{\circ}$ with reasonable beam quality.

\section{LASER PULSE REPITITION FREQUENCY ENHANCER}

The notion to use a LADAR to identify targets requires increased spatial resolution. A capability is needed to increase the number of pixels on the target, which can be accomplished by increasing the scanning time and/or the laser Pulse Repetition Frequency (PRF). As laser scanning rates increase, faster pulse repetition rates are required to ensure at least one laser pulse per pixel in fast beam steering applications. A set of requirements for a low-cost, laser pulse repetition rate enhancer to ensure at least one laser pulse per pixel in fast beam steering applications, was devised. The laser pulse repetition rate enhancer must be capable of supporting high speed scanning in the $\mathrm{kHz}$ to $\mathrm{MHz}$ range.

The increase in PRF of the laser poses tremendous challenges due to the trade-off between the pulse repetition rate and the energy in each pulse. These challenges are overcome via a novel optical phased array technique for low grating lobe fast speed beam steering. ${ }^{15}$ The advanced PRF enhancer, which has been developed in the laboratory, is capable of supporting high speed scanning in the $\mathrm{kHz}$ to $\mathrm{MHz}$ range in support of future LADAR Seeker development project. The laser pulse repetition rate is increased to the $\mathrm{MHz}$ range using unequally spaced optical phased arrays, which is a 4-fold increase from the conventional rate. ${ }^{15}$ A laboratory-version prototype unit has been assembled.

\section{SUMMARY}

A status review of AMRDEC's research and development project to develop Micro Electro-Mechanical Systems (MEMS)-based phased arrays to provide rapid beam steering for sensors, optical and Radio Frequency (RF) missile seekers, and RF communication links has been provided. The inherent low-loss capabilities of MEMS devices make them an attractive candidate for phased arrays to replace bulky gimbals in missile seekers. The technical challenges for the design of MEMS-based phased arrays for RF seekers and communication links, as well as micro lens arrays for LADAR seekers are seen in the areas of reliability, packaging, and low-actuating voltages. The design, fabrication and testing of optical and RF phased array components including micro lens arrays, actuators, MEMS switches, coplanar waveguides and RF phase shifters are demonstrated to overcome the technical barriers for rapid beam steering.

Critical areas of development include actuators to "steer" the optical and RF energy and fabrication processes to ensure device functionality, optimal dielectric materials for device structures, on-wafer packaging techniques, optimal switch geometry, and optimal metal contact to provide good surfaces to reduce insertion loss and increase isolation. Problems such as stiction are overcome with improved design of dielectric materials. Piezoelectrics and various alloys are considered to address the problem of metal contact force and degradation. Hermetic packaging is considered to solve the moisture failure mechanism.

In the case of RF MEMS-based phased arrays, it is found that a 2 bit phase shifter can be used in a phased array (2-inch aperture encompassing 28 antenna or phased element) for a Ku-band communication link to provide directional beam steering to reduce the susceptibility to jamming, as well as provide for a longer communications range with lower transmitted power. In addition, a 3 bit phase shifter can be used in a phased array (6-inch aperture encompassing 1,000 antenna or phase elements) for a Ka-band missile 
seeker. Significant milestones have been achieved via demonstrating RF beam steering achieving $+/-30^{\circ}$ in one design and $+55^{\circ}$ in the second design.

In the case of optical MEMS-based phased arrays, it is found that higher aberration and lower coherence occur at larger beam angles. In addition, larger diameter micro-lenses, which are more difficult to implement, improve diffraction efficiency and coherence of the system. Two significant milestones have been attained. A $+/-25^{\circ}$ field-of-regard was achieved with reasonable beam quality via the demonstration of decentered lens laser beam steering. Finally, a prototype Pulse Repetition Frequency Enhancer, which is capable of supporting high speed scanning in the $\mathrm{kHz}$ to $\mathrm{MHz}$ range, has been demonstrated in the laboratory. This new capability, which is a 4-fold increase from the conventional rate, can support future LADAR Seeker development projects for tracking faster moving targets and enabling faster cruising speeds for missiles or unmanned aerial vehicles.

\section{ACKNOWLEDGEMENTS}

The author wishes to thank the principal investigators (Mr. James C. Holt, Mr. James H. Mullins, Dr. Tracy Hudson, and Ms. Janice Rock) of the AMRDEC research and development project and the entire team of scientists and engineers for their technical contributions. In addition, the technical contributions from Dr. Edward Watson, Dr. S. Yin, and Mr. Chris Heaton are also acknowledged. This paper, which provides a status review of the AMRDEC research and development project on rapid beam steering, emphasizes valuable information from high quality papers (referenced throughout this paper) written by the principal investigators and the Government/industry team.

\section{REFERENCES}

1. K. Sata, K. Nishikawa, and T. Hirako, "Development and Field Experiments of Phased Array Antenna for Land Vehicle Satellite Communications," Antennas and Propagation Society International Symposium, 1992 Digest, Vol. 2, p. 1073-1076, 18-25 July 1992.

2. N. Fourikis, Phased Array-Based Systems and Applications, John Wiley Sons Inc., 1997.

3. P. B. Ruffin and S. Burgett, "Recent Progress in MEMS Technology Development for Military Applications," SPIE Proceedings, Vol. 4334, p. 1-12, March 2001.

4. Website URL http://www.fhr.fgan.de/fhr/fhr_c121_f2 en.html

5. P. B. Ruffin, “Optical MEMS-Based Arrays," SPIE Proceedings, p. 230-241, March 2003.

6. S. A. Hovanessian, Introduction to Sensor System, Artech House, MA, 1988.

7. H. J. De Los Santos, Introduction to Microelectromechanical (MEM) Microwave Systems, Artech House, Boston - London, 1999.

8. G. M. Rebeiz, J. B. Muldavin, "RF MEMS Switches and Switch Circuits," IEEE Microwave Magazine, Vol. 2, No. 4, pp. 59-71, December 2001.

9. T. Campbell, "MEMS Switch Technology Approaches the Ideal Switch," Applied Microwave and Wireless, Vol. 13. No. 5, pp. 100-107, May 2001.

10. S. Cass, "Large Jobs for Little Devices," IEEE Spectrum, Vol. 38, No. 1. pp. 72-73, January 2001.

11. J. H. Mullins, "Candidate RF MEMS Applications for Missile Seekers, RADARS and Communications," TR-RD-MG-03-99, US Army AMRDEC, June 2003.

12. J. C. Rock, J. H. Mullins, and P. B. Ruffin, "Low-Cost Phased Arrays for Tactical Seekers," Proceedings of IEEE Aerospace Symposium, October 2003.

13. E. A. Watson, "Analysis of Beam Steering with Decentered Microlens Arrays," Optical Engineering, Vol. 32, No. 11, p. 2665-2670, November 1993.

14. C. Heaton, P. B. Ruffin, E. A. Watson, and J. C. Holt, "Lithography and Manufacturing Analysis and Considerations in Micro Lens Arrays (MLA) used in Beam Steering Applications," SPIE Proceedings, June 2006.

15. S. Yin, J. Kim, and P. B. Ruffin, "A Fast-Speed Beam Scanner Based on Optical Phased Array,” SPIE Proceeding, Vol. 5904, No. 04, August 2005. 